\title{
Antrochoanal Polyp Harboring Cholesterol Granuloma: A Case Study
}

\section{Osman $\mathrm{NSAA}^{* 1}$ and Haq $\mathrm{ME}^{2}$}

${ }^{1}$ Department of Pathology, Faculty of Medicine, Minia University, Egypt

${ }^{2}$ King Faisal Hospital, Taif, Kingdom of Saudi Arabia

*Corresponding author: Osman NSAA, Department of Pathology, Faculty of Medicine, Minia University, Egypt, E-mail: nisr20032000@yahoo.com

Citation: Osman NSAA, Haq ME (2017) Antrochoanal Polyp Harboring Cholesterol Granuloma: A Case Study. J Case Rep Stud 5(6): 604

Received Date: November 16, 2017 Accepted Date: December 27, 2017 Published Date: December 29, 2017

\begin{abstract}
Cholesterol granuloma (CG) is a foreign body reaction to the deposition and inadequate drainage of cholesterol crystals that are frequently found in association with chronic diseases. The clinical symptoms are non-specific. The differential diagnosis of CG includes cysts, mucocoeles and neoplasms. Radiological and histopathological findings are essential for diagnosis of CG. In this report it is aimed to introduce a case of CG in antrochoanal polyp with its clinical and histopathological findings.
\end{abstract}

Keywords: Antrochoanal polyp; Cholesterol Granuloma; Histopathology

\section{Introduction}

Antrochoanal polyp arises from maxillary sinus, passes through the sinus ostia to the choana. It represents 4-6\% of all nasal polyps and is usually unilateral [1]. Cholesterol granuloma is a pathological lesion usually affects middle ear and temporal bone. It results from a foreign body reaction to cholesterol crystals resulting in granuloma formation. These crystals are believed to be the result of necrosis of fat, blood or tissue products. Cholesterol granuloma is usually unilateral and affect young age.

Histologically, it consists of cholesterol clefts surrounded by foreign body giant cells, foam cells and hemosiderin laden macrophages. It is treated by either surgical removal or conservative steroid therapy.

\section{Case report}

This is a case report of cholesterol granuloma in right antrochoanal polyp in Saudi male patient aged 50 years old. The patient came to outpatient ENT clinic of king Faisal medical complex hospital in Taif, Saudi Arabia; complaining of persistent obstruction in right nasal cavity.

Clinical examination prior to operation showed a polypoid mass obstructing right nasal cavity. Excision biopsy was done. Gross examination showed multiple shiny gray-white polypoid pieces of tissue measuring $5 \mathrm{x} 5 \mathrm{~cm}$ in aggregate. The tissue was fixed

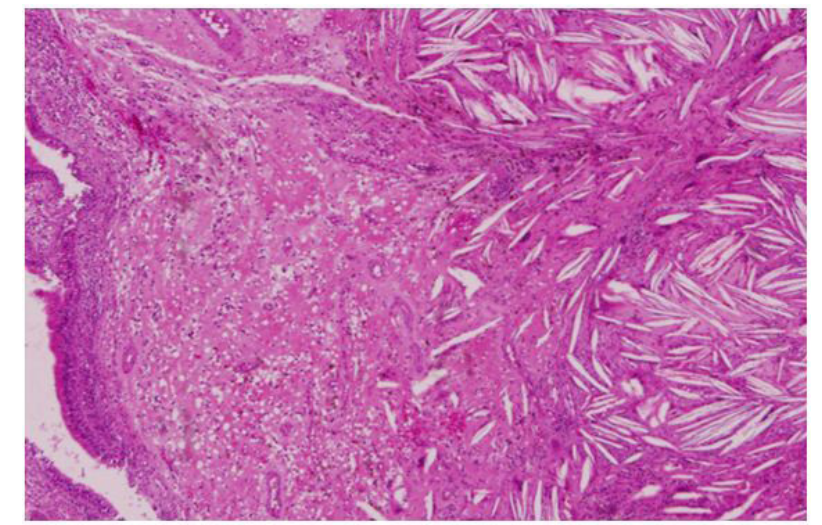

Figure 1: Histopathological analysis revealed polypoid lesion covered by respiratory epithelium with the core containing cholesterol clefts in an oedematous stroma with chronic inflammatory cells (H\&E 10X) 


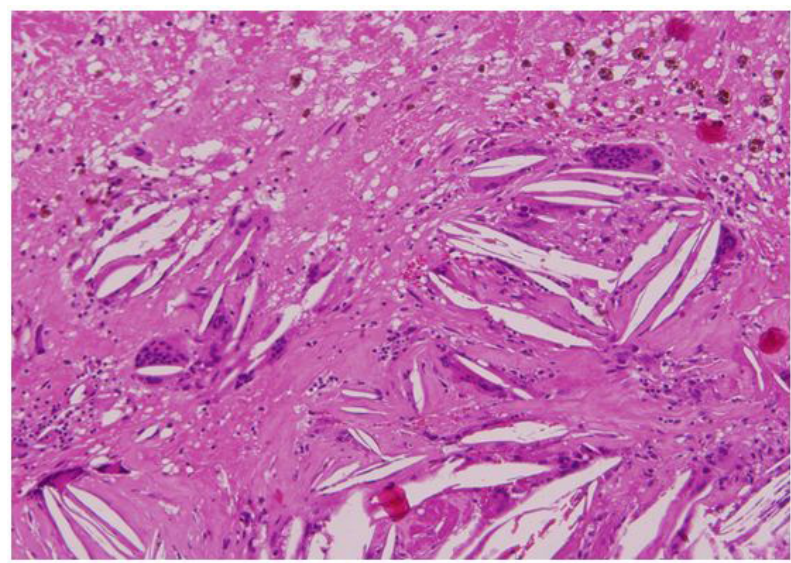

Figure 2: Histopathological analysis revealed cholesterol granuloma: granulation tissue contains multinucleated giant cells, rhomboids of cholesterol crystals in the cells, hemosiderin laden macrophages and chronic inflammatory cells (H\&E 40X)

in $10 \%$ buffered formalin, processed routinely, cut at $5 \mu$ thickness and stained with hematoxylin-eosin (H\&E). Microscopic examination shows polypoid pieces of tissue lined by pseudostratified columnar ciliated epithelium. The core contains numerous chronic inflammatory cells formed of eosinophils, lymphocytes and plasma cells in an edematous stroma (Figure 1). There is multiple cholesterol granulomas formed of cholesterol clefts surrounded by multinucleated giant cells of foreign body type and hemosiderin pigments (Figure 2). No evidence of tumor was seen.

\section{Discussion}

Cholesterol granuloma results from a foreign body reaction to cholesterol crystals precipitated from blood stagnation after allergy, trauma or chronic infection. These Cholesterol crystals resist absorption by giant cells [2]. It usually affects middle-aged men. The etiology of CG is still unclear; many theories postulated impaired drainage, ventilation disturbances and bleeding followed by impaired lymphatic drainage to form CG [3].

Cholesterol granuloma is uncommon in frontal [4] and maxillary sinuses [5]. It was reported in antrochoanal polyps [1]. Cholesterol granulomas are also found outside the head and neck regions; in the breast, the testes, the lungs, the peritoneum and the kidneys [6]. A case of large extradural collection of fluid due to cholesterol granuloma was reported [7]. These lesions usually are discovered incidentally during surgery [8] but it can be suspected clinically by presence of clear golden yellow rhinorrhea as a specific sign [5].

Regarding radiological findings, CG gives high signal intensity in both T1 and T2 magnetic resonance imaging and does not demonstrate change with gadolinium injection [9] (Figure 3). Computerized tomography is essential to localize its bony extension (Figure 4). Cholesterol granuloma is only confirmed by histological examination of the tissue. Histopathologically, there is chronic inflammatory granulation tissue containing large numbers of rhomboid clefts of cholesterol crystals surrounded by foreign body giant cells. Foamy histiocytes and plasma cells are present in large percentage. Fibrin deposition, focal bleeding and hemosiderin are often histological features [10]. Electron microscopic findings include cholesterol needles in the giant cells surrounded by mitochondria, lysosomes and pools of blood corpuscles [2]. Treatment of cholesterol granuloma is either conservative or surgical. Steroid therapy for mild cases is enough in most of cases. Surgical intervention is the gold standard treatment. Complete removal is advised to avoid recurrence after surgery [10]. This raises the demand of endoscopic sinus surgery for initial diagnosis and treatment of this lesion. As for our patient, he underwent simple polypectomy as it was done for diagnostic purpose because of long period with medical treatment for nasal obstruction without effect.

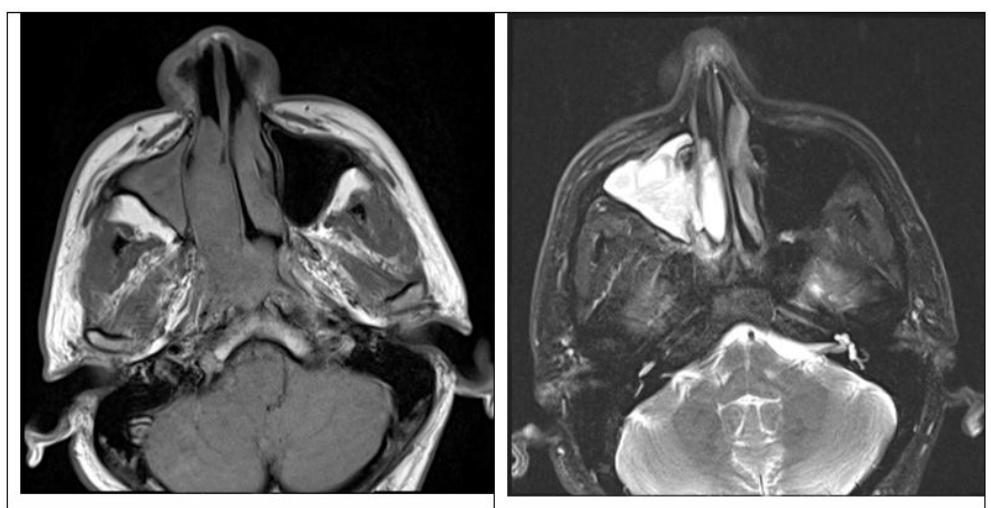

Figure 3: MRI of antrochoanal polyp; The right maxillary sinus, posterior right nasal canal and nasopharynx are occupied with soft tissue. The tissue is T1 isointense and intensely T2 hyperintense with heterogenous enhancement 


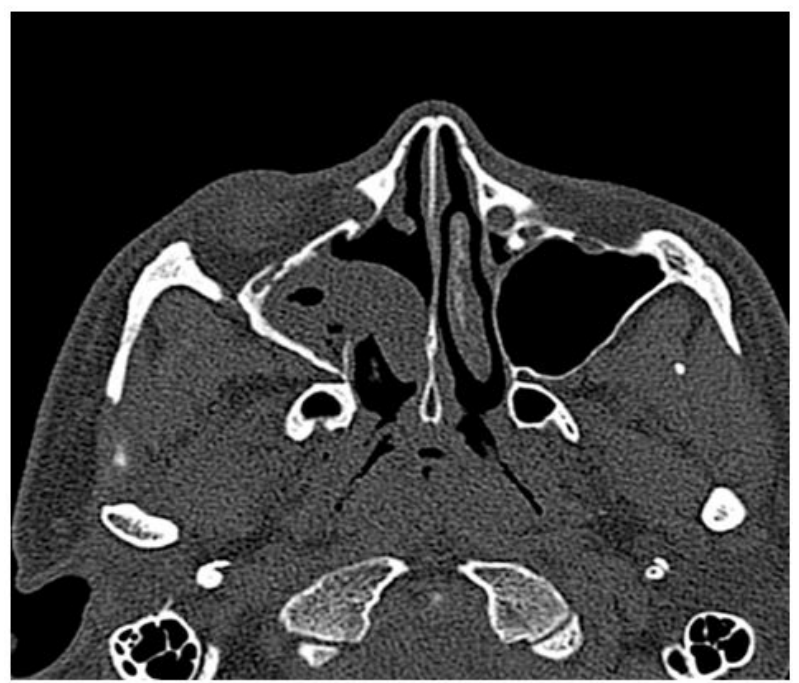

Figure 4: CT of antrochoanal polyp; Soft tissue in the right maxillary sinus extending into nasal canal and nasopharynx with widening of the bony ostium ostium of the maxillary sinus

\section{Conclusion}

Whenever with persistence of nasal infection and obstruction in spite of proper medical treatment; physician should think of cholesterol granuloma.

\section{References}

1. Shvili I, Hadar T, Shvero J, Feinmesser R, Koren R (2005) Cholesterol granulomas in antrochoanal polyps: A clinicopathologic study. Eur Arch Otorhinolaryngol 262: 821-5.

2. Friedmann I, Graham MD (2002) The ultrastructure of cholesterol granuloma of the middle ear: an electron microscopic study. J Laryngol Otol 116: 877-81.

3. Ko MT, Hwang CF, Kao YF, Lui CC, Huang CC, et al. (2006) Cholesterol granuloma of the maxillary sinus presenting as sinonasal polyp. Am J Otolaryngol 27: $370-2$.

4. Shykhon ME, Trotter MI, Morgan DW, Reuser TT, Henderson MJ (2002) Cholesterol granuloma of the frontal sinus. J Laryngol Otol 116: 1041-3.

5. Chao TK (2006) Cholesterol granuloma of the maxillary sinus. Eur Arch Otorhinolaryngol 263: 592-7.

6. Bella Z, Torkos A, Tiszlavicz L, Iván L, Jóri J, et al. (2005) Cholesterol granuloma of the maxillary sinus resembling an invasive destructive tumor. Eur Arch Otorhinolaryngol 262: 531-3.

7. Brodie SW, Chaurasia MK (1985) A rare intracranial complication of cholesterol granuloma. J Laryngol Otol 99: 491-5.

8. Brackmann DE, Toh EH (2002) Surgical management of petrous apex cholesterol granuloma. Otol Neurotol 23: 529-33.

9. Mosnier I, Cyna-Gorse F, Grayeli AB, Fraysse B, Martin C, et al. (2002) Management of cholesterol granulomas of the petrous apex based on clinical and radiologic evaluation. Otol Neurotol 23: 522-8.

10. Georgalas C, Kania R, Guichard JP, Sauvaget E, Tran Ba Huy P, et al. (2008) Endoscopic transsphenoidal surgery for cholesterol granulomas involving the petrous apex. Clin Otolaryngol 33: 38-42. 